\title{
Simple Analytical Particle and Kinetic Energy Densities for a Dilute Fermionic Gas in a $d$-Dimensional Harmonic Trap
}

\author{
Matthias Brack \\ Institut für Theoretische Physik, Universität Regensburg, D-93040 Regensburg, Germany \\ Brandon P. van Zyl \\ Institute for Microstructural Sciences, National Research Council of Canada, Ottawa, Ontario, K1A OR6
}

(October 24, 2018)

\begin{abstract}
We derive simple analytical expressions for the particle density $\rho(r)$ and the kinetic energy density $\tau(r)$ for a system of noninteracting fermions in a $d$-dimensional isotropic harmonic oscillator potential. We test the Thomas-Fermi (TF, or localdensity) approximation for the functional relation $\tau[\rho]$ using the exact $\rho(r)$ and show that it locally reproduces the exact kinetic energy density $\tau(r)$, including the shell oscillations, surprisingly well everywhere except near the classical turning point. For the special case of two dimensions (2D), we obtain the unexpected analytical result that the integral of $\tau_{T F}[\rho(r)]$ yields the exact total kinetic energy.
\end{abstract}

PACS numbers: 03.65.Sq, 05.30.Fk, 31.15.Ew, 71.10.Ca

The evaporative cooling of dilute (i.e., almost noninteracting) fermionic gases has recently been achieved by Jin and DeMarco at JILA in Colorado [1]. This spectacular experimental milestone has stimulated an enormous effort to explore and understand the properties of these new quantum systems, which can be viewed as the quantum analogue of the Bose-Einstein condensation (BEC) recently observed in ultracold trapped Bose gases. While it is true that the theory of homogeneous dilute Fermi systems is fairly well developed, addressing new experiments probing strongly inhomogeneous systems in regimes far from equilibrium, represents a much greater challenge to theory. To this end, Vignolo et al. 22 have recently used a Green's function method to compute the particle and kinetic energy densities for a system of noninteracting fermions in a one-dimensional (1D) harmonic oscillator potential. In principle such a (quasi)-1D system can be achieved experimentally using state-of-theart magnetic confinement techniques [1]. Owing to the enhanced shell structure found in 1D, Vignolo et al. suggest that these quantum oscillations may be accessible to observation in magnetically trapped gases of fermionic alkali atoms. We give here much simpler analytical results for the more general case of a $d$-dimensional harmonic potential and use them to test their Thomas-Fermi (TF) functional relation.

The work presented in this paper is also applicable to a $2 \mathrm{D}$ electron gas confined to so-called quantum dots [3]. The external confinement potential of these dots is in many cases essentially harmonic. Bhaduri et al. [4] have shown that the inclusion of a short-range two-body interaction may be included via fractional statistics, provided that one uses the TF relation $\tau_{T F}[\rho]$ relevant for $2 \mathrm{D}$.

The method. - We start from a system of noninteracting fermions described by the time-independent Schrödinger equation

$$
\hat{H} \phi_{i}(\mathbf{r})=[\hat{T}+V(\mathbf{r})] \phi_{i}(\mathbf{r})=\epsilon_{i} \phi_{i}(\mathbf{r}),
$$

where $V(\mathbf{r})$ is a local potential to be specified later. The single-particle density matrix can be obtained by an inverse Laplace transform of the Bloch density matrix:

$$
\rho\left(\mathbf{r}, \mathbf{r}^{\prime}\right)=2 \sum_{\epsilon_{i}<E_{F}} \phi_{i}^{*}\left(\mathbf{r}^{\prime}\right) \phi_{i}(\mathbf{r})=\mathcal{L}_{E_{F}}^{-1}\left[\frac{2}{\beta} C\left(\mathbf{r}, \mathbf{r}^{\prime} ; \beta\right)\right],
$$

where the latter is defined by

$$
C\left(\mathbf{r}, \mathbf{r}^{\prime} ; \beta\right)=\sum_{\text {all } i} \phi_{i}^{*}\left(\mathbf{r}^{\prime}\right) \phi_{i}(\mathbf{r}) \exp \left\{-\beta \epsilon_{i}\right\} .
$$

$E_{F}$ is the Fermi energy, the factor 2 accounts for spin. We now use center-of-mass and relative coordinates:

$$
\mathbf{q}=\frac{1}{2}\left(\mathbf{r}+\mathbf{r}^{\prime}\right), \quad \mathbf{s}=\mathbf{r}-\mathbf{r}^{\prime}
$$

so that the local density is $\rho(\mathbf{q})=\left.\rho(\mathbf{q}, \mathbf{s})\right|_{\mathbf{s}=0}$. For the kinetic energy density, we investigate two expressions [5]:

$$
\begin{aligned}
\tau(\mathbf{q}) & =-\frac{\hbar^{2}}{2 m} 2 \sum_{\epsilon_{i}<E_{F}} \phi_{i}^{*}(\mathbf{q}) \nabla^{2} \phi_{i}(\mathbf{q}), \\
\tau_{1}(\mathbf{q}) & =\frac{\hbar^{2}}{2 m} 2 \sum_{\epsilon_{i}<E_{F}}\left|\nabla \phi_{i}(\mathbf{q})\right|^{2} .
\end{aligned}
$$

In the presence of time-reversal symmetry they are simply related by

$$
\tau(\mathbf{q})=\tau_{1}(\mathbf{q})+\frac{1}{2} \frac{\hbar^{2}}{2 m} \nabla^{2} \rho(\mathbf{q}) .
$$

A convenient quantity is their mean,

$$
\xi(\mathbf{q})=\frac{1}{2}\left[\tau(\mathbf{q})+\tau_{1}(\mathbf{q})\right]
$$

which is obtained from the density matrix by

$$
\xi(\mathbf{q})=-\frac{\hbar^{2}}{2 m}\left[\nabla_{s}^{2} \rho(\mathbf{q}, \mathbf{s})\right]_{s=0}
$$


where $\nabla_{s}$ is the gradient with respect to the variable s. Note that all three quantities $\tau(\mathbf{q}), \tau_{1}(\mathbf{q})$, and $\xi(\mathbf{q})$ integrate to the same exact kinetic energy.

We now specialize to an isotropic harmonic oscillator potential in $d$ dimensions:

$$
V(r)=\frac{m}{2} \omega^{2} r^{2}
$$

where $r=\sqrt{x_{1}^{2}+\ldots+x_{d}^{2}}$ is the radial variable. The exact Bloch density matrix for this system is given by [6]

$$
\begin{gathered}
C\left(\mathbf{r}, \mathbf{r}^{\prime} ; \beta\right)=C(q, s ; \beta)=\left(\frac{m \omega}{2 \pi \hbar}\right)^{d / 2} \frac{1}{\sinh ^{d / 2}(\beta \hbar \omega)} \times \\
\exp \left\{-\frac{m \omega}{\hbar}\left[q^{2} \tanh \left(\frac{\beta \hbar \omega}{2}\right)+\frac{s^{2}}{4} \operatorname{ctgh}\left(\frac{\beta \hbar \omega}{2}\right)\right]\right\} .
\end{gathered}
$$

To get the particle and kinetic energy densities, we need to perform inverse Laplace transforms of the above function and its derivatives at $s=0$. For the first exponential factor in (11), we employ the following relation which can be derived from Ref. [7]

$$
\begin{aligned}
\exp \{ & -x \tanh (\beta / 2)\} \\
= & \sum_{n=0}^{\infty}(-1)^{n} L_{n}(2 x) e^{-x}\left\{e^{-n \beta}+e^{-(n+1) \beta}\right\} .
\end{aligned}
$$

This relation holds if $|z|=\left|e^{-\beta}\right|<1$, which is fulfilled since the contour of the inverse Laplace transform integral in the complex $\beta$ plane goes along $\beta=i t+c$ for $t \in(-\infty, \infty)$ with $c>0$. Further analytical progress depends on the dimensionality $d$.

The case $d=2$. - Here we can directly use the following exact Laplace inverse [8]

$$
\mathcal{L}_{\lambda}^{-1}\left[\frac{e^{-n \beta}}{\beta \sinh (\beta)}\right]=2 \sum_{k=0}^{\infty} \Theta[\lambda-(2 k+1)-n] .
$$

When filling $M+1$ oscillator shells, the Fermi energy is

$$
E_{F}=\hbar \omega(M+1+\delta),
$$

with $\delta$ being an infinitesimally small positive number. Combining Eqs. (12), (13) and carefully evaluating the sums over the step functions, we get for the density

$$
\rho(q)=2\left(\frac{m \omega}{\pi \hbar}\right) \sum_{\mu=0}^{M}(M-\mu+1)(-1)^{\mu} L_{\mu}(2 x) e^{-x}
$$

where $x=(m \omega / \hbar) q^{2}$. The kinetic energy density (9) is given, after some suitable manipulations of hyperbolic functions, by the following Laplace inverse:

$$
\begin{aligned}
& \xi(q)=\hbar \omega\left(\frac{m \omega}{\pi \hbar}\right) \times \\
& \mathcal{L}_{E_{F}}^{-1}\left[\frac{1}{\beta} \frac{1}{2 \sinh ^{2}(\beta \hbar \omega / 2)} \exp \{-x \tanh (\beta \hbar \omega / 2)\}\right] .
\end{aligned}
$$

Removing one inverse sinh factor by the identity

$$
\frac{1}{2 \sinh (\beta \hbar \omega / 2)}=\sum_{m=0}^{\infty} e^{-(m+1 / 2) \beta \hbar \omega}
$$

and proceeding as above, we get the final expression for the kinetic energy density:

$\xi(q)=\hbar \omega\left(\frac{m \omega}{\pi \hbar}\right) \sum_{\mu=0}^{M}(M-\mu+1)^{2}(-1)^{\mu} L_{\mu}(2 x) e^{-x}$

The integrals $d^{2} q$ of the densities (15), (18) are readily evaluated using

$$
\int_{0}^{\infty} L_{n}(2 x) e^{-x} d x=(-1)^{n}
$$

and yield the correct results for the number $N$ of particles in $M+1$ filled shells

$$
\int \rho(q) d^{2} q=2 \sum_{\mu=0}^{M}(\mu+1)=M^{2}+3 M+2=N(M),
$$

and for their exact kinetic energy $E_{k i n}(M)$

$$
\begin{aligned}
& \int \xi(q) d^{2} q=\hbar \omega \sum_{\mu=0}^{M}(\mu+1)^{2} \\
& =\frac{1}{6} \hbar \omega\left(2 M^{3}+9 M^{2}+13 M+6\right)=E_{k i n}(M) .
\end{aligned}
$$

Next we investigate the Thomas-Fermi (TF) relation between $\tau$ (or $\tau_{1}$ or $\xi$ ) and $\rho$, which in $2 \mathrm{D}$ is

$$
\tau_{T F}[\rho]=\frac{\hbar^{2}}{2 m} \pi \rho^{2},
$$

see also Eq. (33) below. Inserting Eq. (15) into the righthand side above and integrating, using the orthonormality of the Laguerre polynomials, we find

$$
\int \tau_{T F}[\rho(q)] d^{2} q=\hbar \omega \sum_{\mu=0}^{M}(\mu+1)^{2}=E_{k i n}(M) .
$$

This means that the simple TF functional - without gradient corrections [9] - using the exact density $\rho(q)$ yields the exact quantum-mechanical kinetic energy, which is highly nontrivial and unexpected. The local behavior of $\tau_{T F}[\rho(q)]$ will be examined numerically below.

The cases $d=1$ and $d \geq 3$. - For $d=1$, we have a square root of the sinh factor in the denominator of (11). To handle it, we use the expansion

$$
\begin{aligned}
\sinh ^{1 / 2}(s) & =\frac{1}{\sqrt{2}}\left(e^{s}-e^{-s}\right)^{1 / 2}=\frac{1}{\sqrt{2}} e^{s / 2} \sqrt{1-e^{-2 s}} \\
& =\frac{1}{\sqrt{2}} e^{s / 2}\left\{1-\sum_{m=1}^{\infty} \frac{(2 m-3) ! !}{(2 m) ! !} e^{-2 m s}\right\},
\end{aligned}
$$


which converges for Re $s>0$, and include it as a factor on top of the $d=2$ case. For $d=3$, we have to include its inverse, which has the expansion

$$
\begin{aligned}
& \sinh ^{-1 / 2}(s)=\sqrt{2} e^{-s / 2}\left(1-e^{-2 s}\right)^{-1 / 2} \\
&=\sqrt{2} e^{-s / 2}\left\{1+\sum_{m=1}^{\infty} \frac{(2 m-1) ! !}{(2 m) ! !} e^{-2 m s}\right\} .
\end{aligned}
$$

For $d=4$, we need

$$
\sinh ^{-1}(s)=2\left(e^{s}-e^{-s}\right)^{-1}=2 \sum_{m=0}^{\infty} e^{-(2 m+1) s},
$$

and so on. Using $E_{F}=\hbar \omega(M+d / 2+\delta)$ and proceeding as above for the Laplace inversions, we obtain the general expressions

$$
\begin{aligned}
& \rho(q)=\left(\frac{m \omega}{\pi \hbar}\right)^{d / 2} 2 \sum_{\mu=0}^{M} F_{M-\mu}^{(d)}(-1)^{\mu} L_{\mu}(2 x) e^{-x} \\
& \xi(q)=\hbar \omega\left(\frac{m \omega}{\pi \hbar}\right)^{d / 2} \frac{d}{2} \sum_{\mu=0}^{M} G_{M-\mu}^{(d)}(-1)^{\mu} L_{\mu}(2 x) e^{-x} .
\end{aligned}
$$

The coefficients $F_{\nu}^{(d)}, G_{\nu}^{(d)}$ are given by

$$
\begin{aligned}
& F_{\nu}^{(d)}=\nu+1+\sum_{m=1}^{[\nu / 2]}(\nu+1-2 m) g_{m}^{(d)}, \\
& G_{\nu}^{(d)}=(\nu+1)^{2}+\sum_{m=1}^{[\nu / 2]}(\nu+1-2 m)^{2} g_{m}^{(d)},
\end{aligned}
$$

using (for $m \geq 1$ )

$$
\begin{aligned}
& g_{m}^{(1)}=-(2 m-3) ! ! /(2 m) ! ! \quad \text { with } \quad g_{1}^{(1)}=1 / 2, \\
& g_{m}^{(2)}=0, \quad g_{m}^{(3)}=(2 m-1) ! ! /(2 m) ! ! \\
& g_{m}^{(4)}=1, \quad g_{m}^{(5)}=(2 m+1) ! ! /(2 m) ! !
\end{aligned}
$$

and so on. For even dimensions, $F_{\nu}^{(d)}$ and $G_{\nu}^{(d)}$ can be computed analytically, with $[\nu / 2] \equiv \operatorname{integer}(\nu / 2)$.

Like Eqs. (15), (18), to which Eqs. (27), (28) reduce for $2 \mathrm{D}$, the latter are much simpler for numerical computations than their definitions in terms of the eigenfunctions, which necessitate multiple summations for $d \geq 2$.

In the TF or local-density approximation (LDA), one gets from the potential $V(q)=\left(m \omega^{2} / 2\right) q^{2}=(\hbar \omega / 2) x$ the following local densities

$$
\begin{aligned}
& \rho_{T F}(q)=\frac{4}{d} \frac{1}{\Gamma\left(\frac{d}{2}\right)}\left(\frac{m \omega}{2 \pi \hbar}\right)^{d / 2}(\lambda-x / 2)^{d / 2}, \\
& \tau_{T F}(q)=\frac{4 \hbar \omega}{(d+2)} \frac{1}{\Gamma\left(\frac{d}{2}\right)}\left(\frac{m \omega}{2 \pi \hbar}\right)^{d / 2}(\lambda-x / 2)^{d / 2+1},
\end{aligned}
$$

where $\lambda=E_{F} /(\hbar \omega)$. The TF functional relation between $\tau$ and $\rho$ is then given by

$$
\tau_{T F}[\rho]=\frac{\hbar^{2}}{2 m} \frac{4 \pi d}{(d+2)}\left[\frac{d}{4} \Gamma\left(\frac{d}{2}\right)\right]^{2 / d} \rho^{1+2 / d} .
$$

Our objective now is to study numerically the above relation using the exact densities and to see how well it holds locally as well as globally (i.e., upon integration). We have already seen analytically that its integral yields the exact kinetic energy for $d=2$.

Numerical results. - The following figures show numerical results in units such that $\hbar=\omega=m=1$. We make the following observations:

1. As is well known (cf. also Ref. [2]), the densities $\rho(q)$ and $\tau(q), \tau_{1}(q)$ oscillate around the smooth TF densities (31) and (32), respectively, except near the turning point where the latter go to zero (see Figs. 2, 3).

2. The shell oscillations in the quantities $\tau(q)$ and $\tau_{1}(q)$ are exactly opposite in phase, so that their mean $\xi(q)$ is a smooth function of $q$ that (except near the turning point) closely follows the TF density $\tau_{T F}(q)$ given in (32) (see Fig. 11). This has already been observed long ago [10].

3. The functional $\tau_{T F}[\rho(q)]$ in (33), using the exact densities $\rho(q)$, reproduces locally the exact kinetic energy density $\tau(q)$ surprisingly well, including the shell oscillations, except near the classical turning point and in the far tail region (see Figs. 2, 3).

4. The integral of $\tau_{T F}[\rho(q)]$ over the $d$-dimensional space yields the exact kinetic energy only for $d=2$, see Eq. (23). In the other cases it yields kinetic energies with errors less than one percent for $N \gtrsim 14,100$, and 900 in $d=1,3$, and 4 dimensions, respectively.

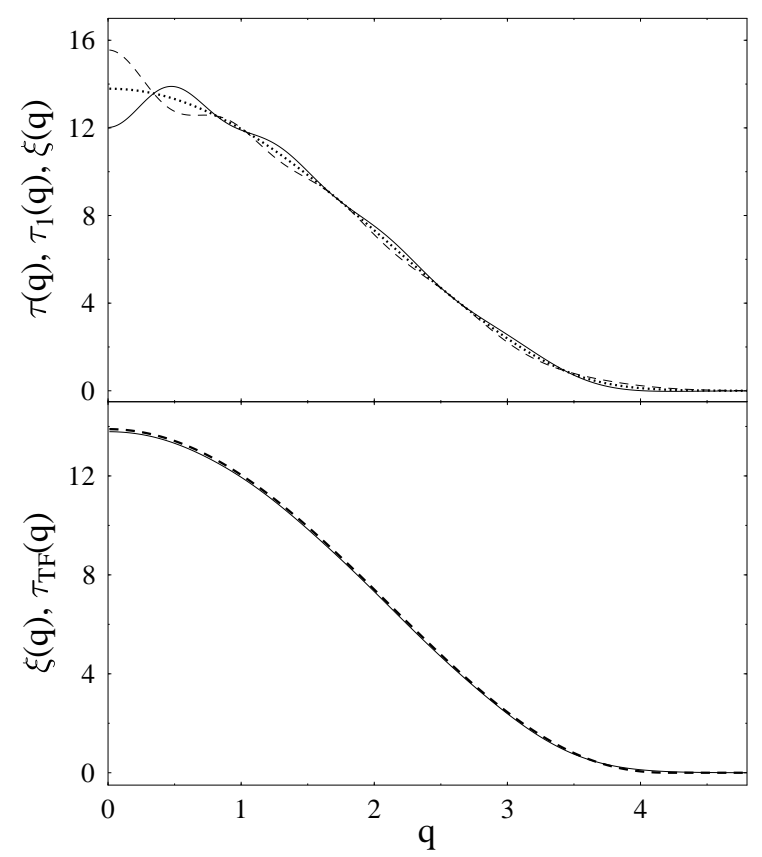

FIG. 1. Kinetic energy densities for $N=240$ particles filling 8 shells of a 3D isotropic harmonic oscillator. Upper panel: solid line: $\tau(q)$, dashed line: $\tau_{1}(q)$, dotted line: $\xi(q)$. Lower panel: solid line: $\xi(q)$, dashed line: $\tau_{T F}(q)$. 


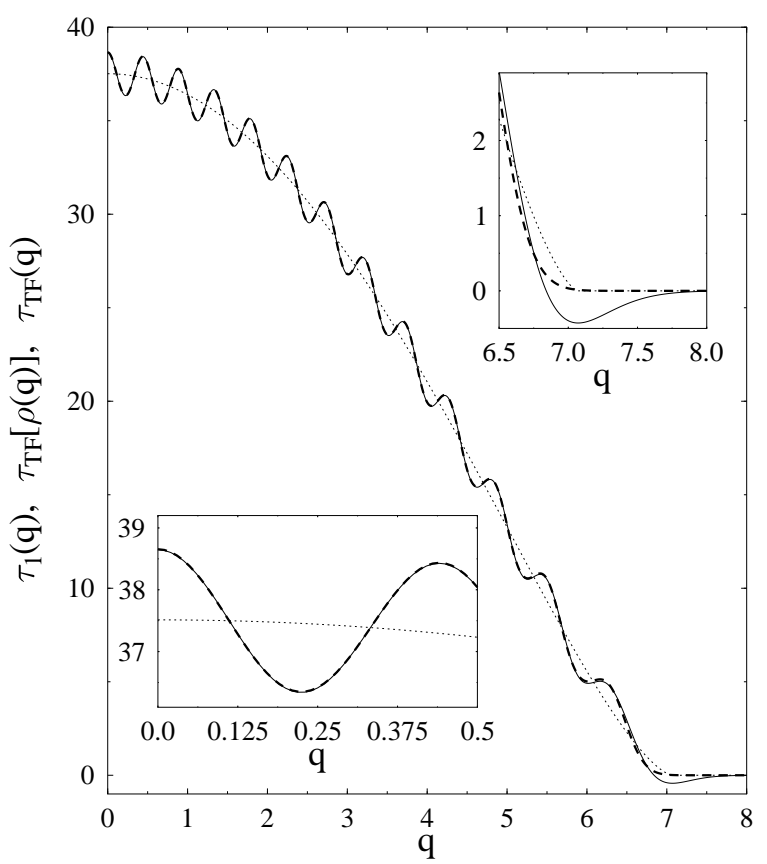

FIG. 2. Kinetic energy densities for $N=50$ particles filling 25 shells of a 1D harmonic oscillator. Solid lines: exact $\tau(q)$. Dashed lines: TF relation $\tau_{T F}[\rho(q)](33)$ using the exact $\rho(q)$. Dotted lines: TF density $\tau_{T F}(q)(32)$. The inserts give close-ups near the center and the tail region.

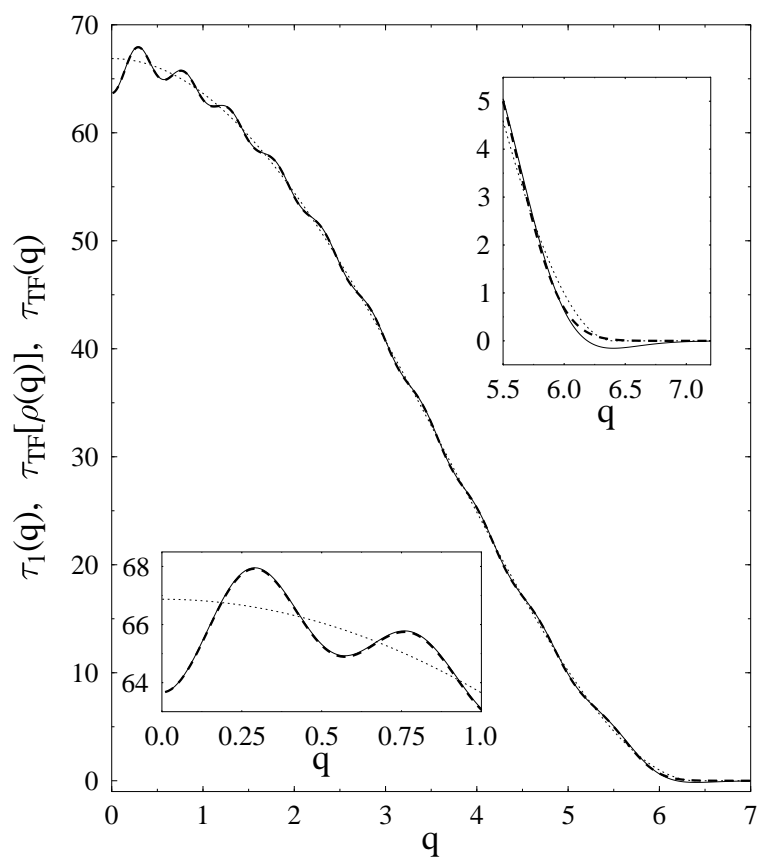

FIG. 3. The same as Fig. 2, but for $N=420$ particles filling 20 shells of a $2 \mathrm{D}$ isotropic harmonic oscillator.

Summary and conclusions. - Our finding that the $\mathrm{TF}$ functional relation $\tau_{T F}[\rho(q)]$ works so well locally is surprising, since it theoretically is exact only in the LDA, i.e., for spatially homogeneous systems. That it reproduces the strong local shell oscillations in $\tau(q)$ so accurately - in the figures, the error cannot been recognized except in the tail regions - is therefore unexpected and does not seem to have been noticed before [11. We must, however, add the caveat that the functional $\tau_{T F}[\rho(q)]$ cannot be variationally exact. As is well known, indeed, the Euler-Lagrange variational equation derived from it leads precisely to the TF density $\rho_{T F}(q)$ in Eq. (31), and not to the exact quantum-mechanical density $\rho(q)$.

In the $2 \mathrm{D}$ case, where the integral even reproduces the exact kinetic energy, our result supports the basic assumptions made in Ref. [- [- concerning the inclusion of a short-range two-body force through fractional statistics, which relies upon the TF relation (22).

Finally, we wish to emphasize that the recent work of Vignolo et al. [2] is a special case of our more general results, and point out that the prominent shell structure displayed in 2D could also become observable in experiments on alkali vapours.

We are grateful to R. K. Bhaduri and M. V. N. Murthy for encouraging discussions and acknowledge the warm hospitality of the Department of Physics and Astronomy at McMaster University. We would also like to acknowledge financial support from the Deutsche Forschungsgemeinschaft and the NSERC of Canda.

[1] B. DeMarco and D. S. Jin, Science 285, 1703 (1999).

[2] P. Vignolo, A. Minguzzi, and M. P. Tosi, Phys. Rev. Lett. 85, 2850 (2000).

[3] see, e.g., L. P. Kouwenhoven, T. H. Oosterkamp, M. W. S. Danoesastro, M. Eto, D. G. Austing, T. Honda, and S. Tarucha, Science 278, 1788 (1997).

[4] R. K. Bhaduri, M. V. N. Murthy, and M. K. Shrivastava, Phys. Rev. Lett. 76, 165 (1996); see also M. K. Shrivastava, R. K. Bhaduri, J. Law, and M. V. N. Murthy, Can. J. Phys. 78, 9 (2000).

[5] In the standard literature on density functional theory, $\tau(\mathbf{q})$ usually denotes the present quantity $\tau_{1}(\mathbf{q})$, Eq. (6).

[6] E. H. Sondheimer and A. H. Wilson, Proc. R. Soc. London Ser. A 210, 173 (1951).

[7] I. S. Gradshteyn and I. M. Ryzhik: Table of Integrals, Series, and Products (Academic Press, New York, 5th edition, 1994), Eq. 8.975.1.

[8] M. Abramowitz and I. A. Stegun: Handbook of Mathematical Functions (Dover Publications, 9th printing, New York, 1970), Eq. 29.3.70.

[9] see, e.g., M. Brack and R. K. Bhaduri: Semiclassical Physics (Frontiers in Physics, Vol. 96) (Addison-Wesley, Reading, 1997). One of us (BVZ) has recently shown that to all orders in $\hbar$, there are no gradient corrections to the kinetic energy density for $d=2$ (unpublished results).

[10] R. K. Bhaduri and L. F. Zaifman, Can. J. Phys. 57, 1990 (1979); C. Guet and M. Brack, Z. Phys. A 297, 247 (1980).

[11] One obtains very similar results also for fermions in a $1 \mathrm{D}$ box and spherical 2D or 3D billiards (M. Brack, unpublished results). 